\title{
QUADRATIC DIOPHANTINE EQUATIONS WITH APPLICATIONS TO QUARTIC EQUATIONS
}

\author{
AJAI CHOUDHRY
}

\begin{abstract}
In this paper, we first show that, under certain conditions, the solution of a single quadratic diophantine equation in four variables $Q\left(x_{1}, x_{2}, x_{3}, x_{4}\right)=0$ can be expressed in terms of bilinear forms in four parameters. We use this result to establish a necessary, though not sufficient, condition for the solvability of the simultaneous quadratic diophantine equations

$$
Q_{j}\left(x_{1}, x_{2}, x_{3}, x_{4}\right)=0, \quad j=1,2,
$$

and give a method of obtaining their complete solution. In general, when these two equations have a rational solution, they represent an elliptic curve, but we show that there are several cases in which their complete solution may be expressed by a finite number of parametric solutions and/or a finite number of primitive integer solutions. Finally, we relate the solutions of the quartic equation

$$
y^{2}=t^{4}+a_{1} t^{3}+a_{2} t^{2}+a_{3} t+a_{4}
$$

to the solutions of a pair of quadratic diophantine equations, and thereby obtain new formulae for deriving rational solutions of the aforementioned quartic equation starting from one or two known solutions.
\end{abstract}

1. Introduction. In this paper, we study the solutions of the single quadratic diophantine equation,

$$
Q\left(x_{1}, x_{2}, x_{3}, x_{4}\right)=0
$$

as well as solutions of a pair of simultaneous quadratic diophantine equations,

$$
Q_{j}\left(x_{1}, x_{2}, x_{3}, x_{4}\right)=0, \quad j=1,2,
$$

2010 AMS Mathematics subject classification. Primary 11D09, 11D25.

Keywords and phrases. Bilinear solutions of quadratic diophantine equations, quartic diophantine equation, quartic model of elliptic curve, quartic function made a perfect square.

Received by the editors on June 14, 2014, and in revised form on August 6, 2014. DOI: $10.1216 / \mathrm{RMJ}^{2}$ 2016-46-3-769 Copyright (C)2016 Rocky Mountain Mathematics Consortium 
where

$$
Q\left(x_{1}, x_{2}, x_{3}, x_{4}\right) \text { and } Q_{j}\left(x_{1}, x_{2}, x_{3}, x_{4}\right), \quad j=1,2 \text {, }
$$

are quadratic forms with rational coefficients in four variables $x_{i}$, $i=1,2,3,4$. We also consider the application of these solutions to the quartic diophantine equation,

$$
y^{2}=t^{4}+a_{1} t^{3}+a_{2} t^{2}+a_{3} t+a_{4},
$$

where the coefficients $a_{i}, i=1,2,3,4$, are rational numbers.

The usual method of solving equation (1.1), as described in [1, page 432], consists of first finding a single numerical solution, and then using it to obtain the complete solution. This complete solution is obtained in terms of polynomials of degree 2 in three parameters. There are very few equations of type (1.1) for which the existing literature gives the complete solution in terms of bilinear forms in four parameters (see, for instance, [2, page 15]). We show that the complete solution of the quadratic diophantine equation (1.1) can be expressed, under certain conditions, in terms of bilinear forms in four variables. For instance, the complete solution of the diophantine equation,

$$
270 x_{1}^{2}+76 x_{1} x_{2}+152 x_{1} x_{3}-16 x_{2}^{2}-48 x_{2} x_{3}-35 x_{3}^{2}+3 x_{4}^{2}=0,
$$

may be expressed as,

$$
\begin{aligned}
& x_{1}=4 p m-2 q n, \quad x_{2}=(117 p+4 q) m+(2 p-65 q) n, \\
& x_{3}=-(72 p+2 q) m-(p-40 q) n, \quad x_{4}=2 q m-p n,
\end{aligned}
$$

where $p, q, m, n$, are arbitrary parameters.

In fact, in Section 2, we obtain a necessary and sufficient condition for the solution of an equation of type (1.1) to be expressed in terms of bilinear forms and give a method of obtaining such solutions.

In Section 3, we obtain a necessary condition for the solvability of the simultaneous equations (1.2) and give a method of obtaining their complete solution when this condition is satisfied. It is well-known that, in general, when the two equations have a rational solution, they represent an elliptic curve. We show, however, that there are numerous cases when the complete solution of these equations may be given by one or more parametric solutions and they may have, in addition, a finite number of primitive solutions. As an example, the complete 
solution of the simultaneous diophantine equations,

$$
\begin{aligned}
x_{1}^{2}-9 x_{2}^{2}-x_{3}^{2}+4 x_{4}^{2} & =0, \\
3 x_{1}^{2}-30 x_{1} x_{2}-4 x_{1} x_{3}-9 x_{2}^{2}-12 x_{2} x_{3} & \\
-7 x_{3}^{2}+12 x_{3} x_{4}+4 x_{4}^{2} & =0,
\end{aligned}
$$

is given by two parametric solutions, a linear solution, namely,

$$
x_{1}=2 m, x_{2}=2 n, x_{3}=-2 m, x_{4}=-3 n,
$$

and a solution of degree 3 ,

$$
\begin{aligned}
& x_{1}=6 m^{2} n-108 m n^{2}+270 n^{3}, \\
& x_{2}=2 m^{3}-8 m^{2} n+18 m n^{2}-36 n^{3}, \\
& x_{3}=30 m^{2} n-36 m n^{2}+270 n^{3}, \\
& x_{4}=3 m^{3}-12 m^{2} n+63 m n^{2}+54 n^{3},
\end{aligned}
$$

where $m, n$ are arbitrary parameters.

In Section 4, we derive formulae giving new solutions of the quartic equation (1.3) starting from one or two known solutions. While there is a vast amount of literature dealing with equation (1.3), the formulae given in Section 4 do not seem to have been obtained earlier. The solutions of (1.3) obtained by these formulae are much simpler than those obtained by existing methods.

With respect to the solutions of homogeneous diophantine equations of type (1.1) or (1.2), we note that the existence of any rational solution

$$
\left(x_{1}, x_{2}, x_{3}, x_{4}\right)=\left(\alpha_{1}, \alpha_{2}, \alpha_{3}, \alpha_{4}\right)
$$

implies that

$$
\left(x_{1}, x_{2}, x_{3}, x_{4}\right)=\left(k \alpha_{1}, k \alpha_{2}, k \alpha_{3}, k \alpha_{4}\right), \quad k \in \mathbb{Q} \backslash\{0\}
$$

is also a rational solution, and all such solutions will be considered equivalent. This equivalence class of solutions will be considered as a single solution of the equation(s) under consideration.

2. The diophantine equation $Q\left(x_{1}, x_{2}, x_{3}, x_{4}\right)=0$. In this section, we first prove a preliminary lemma regarding the diophantine equation

$$
a_{1} x_{1}^{2}+a_{2} x_{2}^{2}+a_{3} x_{3}^{2}+a_{4} x_{4}^{2}=0,
$$


and then prove a theorem that gives a necessary and sufficient condition for the complete solution of this equation to be expressed in terms of bilinear forms in four parameters. We also obtain this bilinear solution explicitly when this condition is satisfied. Next, we prove two theorems that give conditions for the existence of bilinear solutions of the general quadratic equation (1.1) and show how such solutions can be obtained. We also give a couple of examples of bilinear solutions of quadratic equations.

2.1. Bilinear solutions of the equation $Q\left(x_{1}, x_{2}, x_{3}, x_{4}\right)=0$.

Lemma 2.1. If $a_{i}, i=1,2,3,4$, are nonzero rational numbers such that $a_{1} a_{2} a_{3} a_{4}$ is a perfect square, the diophantine equation

$$
a_{1} x_{1}^{2}+a_{2} x_{2}^{2}+a_{3} x_{3}^{2}+a_{4} x_{4}^{2}=0
$$

has a nontrivial solution in integers if and only if the diophantine equation

$$
a_{1} y_{1}^{2}+a_{2} y_{2}^{2}+a_{3} y_{3}^{2}=0
$$

has a nontrivial solution in integers.

Proof. If $a_{1} a_{2} a_{3} a_{4}=k^{2}, k \in \mathbb{Q} \backslash\{0\}$, and equation (2.2) has a nontrivial solution in integers, two of the coefficients $a_{i}$ must be positive and two must be negative. We assume without loss of generality that $a_{1}$ and $a_{2}$ are positive and $a_{3}$ and $a_{4}$ are negative. If $x_{i}=\alpha_{i}, i=1,2,3,4$, is a nontrivial solution of (2.2), we write

$$
\begin{aligned}
& \beta_{1}=a_{2}\left(k \alpha_{2} \alpha_{4}+a_{1} a_{3} \alpha_{1} \alpha_{3}\right), \\
& \beta_{2}=a_{1}\left(k \alpha_{1} \alpha_{4}-a_{2} a_{3} \alpha_{2} \alpha_{3}\right), \\
& \beta_{3}=a_{1} a_{2}\left(a_{3} \alpha_{3}^{2}+a_{4} \alpha_{4}^{2}\right) .
\end{aligned}
$$

We note that $\beta_{3} \neq 0$ since the relation $\beta_{3}=0$ would imply that both $\alpha_{3}$ and $\alpha_{4}$ are 0 , and hence, both $\alpha_{1}$ and $\alpha_{2}$ must also be 0 , contradicting the fact that $x_{i}=\alpha_{i}$ is a nontrivial solution of (2.2). Now, on using the relation $a_{1} a_{2} a_{3} a_{4}=k^{2}$, we get

$$
\begin{aligned}
a_{1} \beta_{1}^{2}+a_{2} \beta_{2}^{2}+a_{3} \beta_{3}^{2} & =a_{1}^{2} a_{2}^{2} a_{3}\left(a_{3} \alpha_{3}^{2}+a_{4} \alpha_{4}^{2}\right)\left(a_{1} \alpha_{1}^{2}+a_{2} \alpha_{2}^{2}+a_{3} \alpha_{3}^{2}+a_{4} \alpha_{4}^{2}\right) \\
& =0 .
\end{aligned}
$$

It follows that $y_{i}=\beta_{i}, i=1,2,3$, is a nontrivial solution of (2.3). Conversely, if $y_{i}=\beta_{i}, i=1,2,3$, is a given nontrivial solution 
of (2.3), a nontrivial solution of (2.2) is given by $\left(x_{1}, x_{2}, x_{3}, x_{4}\right)=$ $\left(\beta_{1}, \beta_{2}, \beta_{3}, 0\right)$. This completes the proof.

Theorem 2.2. If $a_{i}, i=1,2,3,4$, are nonzero rational numbers, the complete solution of a solvable equation of type (2.2) can be expressed in terms of bilinear forms in four parameters if and only if $a_{1} a_{2} a_{3} a_{4}$ is a perfect square. If $a_{1} a_{2} a_{3} a_{4}=k^{2}$ and $x_{i}=\alpha_{i}, i=1,2,3,4$, is a nontrivial solution of equation (2.2) such that $a_{3} \alpha_{3}^{2}+a_{4} \alpha_{4}^{2} \neq 0$, this complete bilinear solution of $(2.2)$ is given by

$$
\begin{aligned}
x_{1}= & \left(a_{1} a_{3} \alpha_{1} \alpha_{3}+k \alpha_{2} \alpha_{4}\right) a_{1} a_{2} a_{4} p r \\
& -\left(a_{2} a_{3} \alpha_{2} \alpha_{3}-k \alpha_{1} \alpha_{4}\right) a_{1} a_{2} a_{4} p s \\
& -\left(a_{2} a_{3} \alpha_{2} \alpha_{3}-k \alpha_{1} \alpha_{4}\right) a_{1} a_{2} a_{4} q r \\
& -\left(a_{1} a_{3} \alpha_{1} \alpha_{3}+k \alpha_{2} \alpha_{4}\right) a_{2}^{2} a_{4} q s, \\
x_{2}= & \left(a_{2} a_{3} \alpha_{2} \alpha_{3}-k \alpha_{1} \alpha_{4}\right) a_{1}^{2} a_{4} p r \\
& +\left(a_{1} a_{3} \alpha_{1} \alpha_{3}+k \alpha_{2} \alpha_{4}\right) a_{1} a_{2} a_{4} p s \\
& +\left(a_{1} a_{3} \alpha_{1} \alpha_{3}+k \alpha_{2} \alpha_{4}\right) a_{1} a_{2} a_{4} q r \\
& -\left(a_{2} a_{3} \alpha_{2} \alpha_{3}-k \alpha_{1} \alpha_{4}\right) a_{1} a_{2} a_{4} q s, \\
x_{3}= & -a_{1} a_{2} a_{4}\left(a_{3} \alpha_{3}^{2}+a_{4} \alpha_{4}^{2}\right)\left(a_{1} p r+a_{2} q s\right), \\
x_{4}= & -a_{1} a_{2} k\left(a_{3} \alpha_{3}^{2}+a_{4} \alpha_{4}^{2}\right)(p s-q r),
\end{aligned}
$$

where $p, q, r$ and $s$ are arbitrary parameters.

Alternatively, if $y_{i}=\beta_{i}, i=1,2,3$, is a solution of equation (2.3) such that $\beta_{3} \neq 0$, the complete bilinear solution of (2.2) may be written as follows:

$$
\begin{aligned}
& x_{1}=a_{4}\left(a_{1} \beta_{1} p+a_{2} \beta_{2} q\right) r-a_{2} a_{4}\left(-\beta_{2} p+\beta_{1} q\right) s, \\
& x_{2}=a_{1} a_{4}\left(-\beta_{2} p+\beta_{1} q\right) r+a_{4}\left(a_{1} \beta_{1} p+a_{2} \beta_{2} q\right) s, \\
& x_{3}=-a_{4} \beta_{3}\left(a_{1} p r+a_{2} q s\right), \\
& x_{4}=k \beta_{3}(q r-p s)
\end{aligned}
$$

where, as before, $p, q, r$ and $s$ are arbitrary parameters.

Proof. If $a_{1} a_{2} a_{3} a_{4}=k^{2}, k \neq 0$, and equation (2.2) is solvable, it follows from Lemma 2.1 that equation (2.3) necessarily has a nontrivial solution, and we may take this solution as $y_{i}=\beta_{i}, i=1,2,3$, with $\beta_{3} \neq 0$. We will now obtain the complete solution of (2.2) by using the 
linear transformation given by

$$
\left[\begin{array}{llll}
x_{1} & x_{2} & x_{3} & x_{4}
\end{array}\right]=\left[\begin{array}{cccc}
a_{1} a_{4} \beta_{1} & -a_{2} a_{4} \beta_{1} & a_{2} a_{4} \beta_{2} & a_{2} a_{4} \beta_{2} \\
-a_{1} a_{4} \beta_{2} & a_{2} a_{4} \beta_{2} & a_{1} a_{4} \beta_{1} & a_{1} a_{4} \beta_{1} \\
-a_{1} a_{4} \beta_{3} & -a_{2} a_{4} \beta_{3} & 0 & 0 \\
0 & 0 & -k \beta_{3} & k \beta_{3}
\end{array}\right]\left[\begin{array}{c}
X_{1} \\
X_{2} \\
X_{3} \\
X_{4}
\end{array}\right] .
$$

As the determinant of the matrix of this linear transformation works out to $4 k a_{1} a_{2} a_{4}^{3} \beta_{3}^{2}\left(a_{1} \beta_{1}^{2}+a_{2} \beta_{2}^{2}\right)$, which is not zero since $\beta_{3} \neq 0$, the linear transformation (2.7) is invertible. Applying this transformation, equation (2.2) becomes

$$
\begin{gathered}
\left.a_{4}\left\{\left(a_{1} X_{1}-a_{2} X_{2}\right)^{2}+a_{1} a_{2}\left(X_{3}+X_{4}\right)^{2}\right)\right\}\left(a_{1} \beta_{1}^{2}+a_{2} \beta_{2}^{2}\right) \\
+\left\{a_{1}^{2} a_{3} a_{4} X_{1}^{2}+2 a_{1} a_{2} a_{3} a_{4} X_{1} X_{2}+a_{2}^{2} a_{3} a_{4} X_{2}^{2}\right. \\
\left.+k^{2}\left(X_{3}-X_{4}\right)^{2}\right\} \beta_{3}^{2}=0 .
\end{gathered}
$$

Using the relations $\beta_{3}^{2}=-\left(a_{1} \beta_{1}^{2}+a_{2} \beta_{2}^{2}\right) a_{3}$ (which is a consequence of $y_{i}=\beta_{i}, i=1,2,3$, being a solution of (2.3)), and $k^{2}=a_{1} a_{2} a_{3} a_{4}$, equation (2.8) reduces to

$$
-4 a_{1} a_{2} a_{4}\left(a_{1} \beta_{1}^{2}+a_{2} \beta_{2}^{2}\right)\left(X_{1} X_{2}-X_{3} X_{4}\right)=0,
$$

where we note that $4 a_{1} a_{2} a_{4}\left(a_{1} \beta_{1}^{2}+a_{2} \beta_{2}^{2}\right) \neq 0$. Thus, we get $X_{1} X_{2}-$ $X_{3} X_{4}=0$, and as is well-known [3, page 69], the complete solution of this equation is given by

$$
X_{1}=p r, \quad X_{2}=q s, \quad X_{3}=p s, \quad X_{4}=q r .
$$

With these values of $X_{i}$, the complete solution of (2.2) is given by (2.7). This is a bilinear solution which is given explicitly by (2.6). If $x_{i}=\alpha_{i}, i=1,2,3$, is a nontrivial solution of equation (2.2) such that $a_{3} \alpha_{3}^{2}+a_{4} \alpha_{4}^{2} \neq 0$, we may take the values of $\beta_{i}, i=1,2,3$, as defined by (2.4) and, substituting these values in (2.6), we get the bilinear solution (2.5).

We will now prove that, if equation (2.2) has a bilinear solution in four parameters, then $a_{1} a_{2} a_{3} a_{4}$ must necessarily be a perfect square. If equation (2.2) has such a bilinear solution, it also has a linear solution in terms of two independent parameters, and therefore, without loss of generality, we may take $x_{1}$ and $x_{2}$ as arbitrary and write this solution as

$$
x_{3}=f_{1} x_{1}+f_{2} x_{2} \quad \text { and } \quad x_{4}=f_{3} x_{1}+f_{4} x_{2},
$$


where $f_{i}, i=1,2,3,4$, are rational numbers. We note that both $f_{1}$ and $f_{2}$ cannot be 0 . Substituting these values of $x_{3}$ and $x_{4}$ in (2.2), we get

$$
\begin{aligned}
&\left(a_{1}+f_{1}^{2} a_{3}+f_{3}^{2} a_{4}\right) x_{1}^{2}+(\left.2 f_{1} f_{2} a_{3}+2 f_{3} f_{4} a_{4}\right) x_{1} x_{2} \\
&+\left(a_{2}+f_{2}^{2} a_{3}+f_{4}^{2} a_{4}\right) x_{2}^{2}=0 .
\end{aligned}
$$

Since (2.11) is identically true for all values of $x_{1}$ and $x_{2}$, the coefficients of $x_{1}^{2}, x_{1} x_{2}$ and $x_{2}^{2}$ in (2.11) must be 0 , and we thus get three equations which can be solved for $a_{1}, a_{2}$ and $a_{3}$. If $f_{1}$ and $f_{2}$ are both nonzero, the values of $a_{1}, a_{2}$ and $a_{3}$ lead to the relation

$$
a_{1} a_{2} a_{3} a_{4}=a_{4}^{4}\left(f_{1} f_{4}-f_{2} f_{3}\right)^{2} f_{3}^{2} f_{4}^{2} /\left(f_{1}^{2} f_{2}^{2}\right) .
$$

If $f_{1}=0$, then for equation (2.11) to be identically satisfied for all $x_{1}$ and $x_{2}$, we must necessarily also have $f_{4}=0$ and we then get

$$
a_{1}=-f_{3}^{2} a_{4}, \quad a_{2}=-f_{2}^{2} a_{3},
$$

so that

$$
a_{1} a_{2} a_{3} a_{4}=f_{2}^{2} f_{3}^{2} a_{3}^{2} a_{4}^{2} .
$$

Similarly, if $f_{2}=0$, we get $f_{3}=0, a_{1}=-f_{1}^{2} a_{3}, a_{2}=-f_{4}^{2} a_{4}$ and so

$$
a_{1} a_{2} a_{3} a_{4}=f_{1}^{2} f_{4}^{2} a_{3}^{2} a_{4}^{2} .
$$

Thus, in all cases, $a_{1} a_{2} a_{3} a_{4}$ is a perfect square. This completes the proof.

Theorem 2.3. If $Q\left(x_{1}, x_{2}, x_{3}, x_{4}\right)$ is a quadratic form, with rational coefficients, in four independent variables $x_{1}, x_{2}, x_{3}, x_{4}$, the matrix of the form $Q\left(x_{i}\right)$ is $A$, and the diophantine equation

$$
Q\left(x_{1}, x_{2}, x_{3}, x_{4}\right)=0,
$$

has a solution in integers, the complete solution of (2.13) can be expressed in terms of bilinear forms in four variables if and only if the determinant $|A|$ is a nonzero perfect square.

Proof. It is well-known that any rational quadratic form in $n$ variables can be reduced by an invertible rational linear transformation to a diagonal form. Denoting the column vectors

$$
\left\{x_{1}, x_{2}, x_{3}, x_{4}\right\} \text { and }\left\{y_{1}, y_{2}, y_{3}, y_{4}\right\}
$$


by $X$ and $Y$, respectively, the transpose of any matrix $M$ by $M^{\prime}$, and the invertible linear transformation that reduces the quadratic form $Q\left(x_{1}, x_{2}, x_{3}, x_{4}\right)$ to a diagonal form by $X=P Y$, we have $|P| \neq 0$, and

$$
P^{\prime} A P=\operatorname{diag}\left[\begin{array}{llll}
a_{1} & a_{2} & a_{3} & a_{4}
\end{array}\right],
$$

where $a_{i} \in \mathbb{Q} \backslash\{0\}$ (since the form $Q\left(x_{1}, x_{2}, x_{3}, x_{4}\right)$ has four independent variables), while equation (2.13) reduces to the equation

$$
a_{1} y_{1}^{2}+a_{2} y_{2}^{2}+a_{3} y_{3}^{2}+a_{4} y_{4}^{2}=0 .
$$

It follows from (2.14) that $\left|P^{\prime}\right| \cdot|A| \cdot|P|=a_{1} a_{2} a_{3} a_{4}$, and so,

$$
|P|^{2} \cdot|A|=a_{1} a_{2} a_{3} a_{4} .
$$

If the diophantine equation (2.13) is solvable, its complete solution can be expressed in terms of bilinear forms in four variables if and only if the complete solution of equation (2.15) can be so expressed. By Theorem 2.2, this is possible if and only if $a_{1} a_{2} a_{3} a_{4}$ is a nonzero perfect square, and the theorem now follows readily from (2.16).

A bilinear solution of equation (2.13) may be obtained by reducing it, using a suitable linear transformation $X=P Y$, to an equation of type (2.15), obtaining a bilinear solution of (2.15) by Theorem 2.2, and finally using the relations $X=P Y$.

We note en passant that it follows from (2.16) that $|A|=0$ if and only if the form $Q\left(x_{1}, x_{2}, x_{3}, x_{4}\right)$ has fewer than four independent variables. When $|A|=0$, at least one of the $a_{i}$ must be 0 . If only one of the four rational numbers $a_{i}$ is 0 , then equation (2.15), and so also equation (2.13), cannot have a linear solution in two independent parameters. If two of these four numbers, say $a_{3}$ and $a_{4}$, are 0 and $-a_{1} a_{2}$ is a perfect square, it is readily seen that both equation (2.15) and equation (2.13) have a linear solution in three independent parameters, and the same is true if three of the four numbers $a_{i}$ are 0 .

We now prove a theorem which shows that, even under the weaker assumption that equation (2.13) has a solution in linear forms in two variables, it is true that $|A|$ is a nonzero perfect square.

Theorem 2.4. If $Q\left(x_{1}, x_{2}, x_{3}, x_{4}\right)$ is a quadratic form, with rational coefficients, in four independent variables $x_{1}, x_{2}, x_{3}, x_{4}$, the matrix 
of the form $Q\left(x_{i}\right)$ is $A$ and the diophantine equation (2.13) has a solution in terms of linear forms in two independent variables, then $|A|$ is a nonzero perfect square and the complete solution of (2.13) can be expressed in terms of bilinear forms in four variables.

Proof. As in Theorem 2.3, we apply an invertible linear transformation $X=P Y$ to reduce equation (2.13) to the diagonal form (2.15), and further, we have $|P|^{2} \cdot|A|=a_{1} a_{2} a_{3} a_{4}$. Equation (2.15) will also have a solution in terms of linear forms in two independent variables, and since we have already shown in the last part of the proof of Theorem 2.2 that, in such a situation, $a_{1} a_{2} a_{3} a_{4}$ must be a nonzero perfect square, it follows that $|A|=a_{1} a_{2} a_{3} a_{4}|P|^{-2}$ must also be a nonzero perfect square. It now follows from Theorem 2.3 that the complete solution of (2.13) can be expressed in terms of bilinear forms in four variables.

2.2. Two numerical examples. We now give two numerical examples illustrating the application of the results of subsection 2.1. As a simple example, we consider the quadratic equation

$$
x_{1}^{2}-9 x_{2}^{2}-x_{3}^{2}+4 x_{4}^{2}=0,
$$

which has a solution $\left(x_{1}, x_{2}, x_{3}, x_{4}\right)=(1,0,1,0)$, and the product $a_{1} a_{2} a_{3} a_{4}$ is a perfect square, namely 36 , so the conditions of Theorem 2.2 are satisfied. Using (2.5) and replacing $r$ and $s$, respectively, by $m / 18$ and $n / 18$, respectively, we obtain the following complete solution of (2.17):

$$
\begin{aligned}
& x_{1}=2 p m+18 q n, \quad x_{2}=2 p n+2 q m, \\
& x_{3}=-2 p m+18 q n, \quad x_{4}=-3 p n+3 q m,
\end{aligned}
$$

where $p, q, m$ and $n$ are arbitrary parameters.

As a second example, we consider equation (1.4), which has a solution $\left(x_{1}, x_{2}, x_{3}, x_{4}\right)=(0,2,-1,-1)$, and the determinant of its matrix $A$ works out to 36 , which is a perfect square. Applying the linear transformation,

$$
\begin{array}{ll}
x_{1}=y_{1}-y_{2}+y_{3}, & x_{2}=31 y_{1}-29 y_{2}+26 y_{3}, \\
x_{3}=-19 y_{1}+18 y_{2}-16 y_{3}, & x_{4}=y_{4},
\end{array}
$$


reduces equation (1.4) to the diagonal form,

$$
y_{1}^{2}+2 y_{2}^{2}-6 y_{3}^{2}-3 y_{4}^{2}=0 .
$$

Equation (2.20) has a solution $\left(y_{1}, y_{2}, y_{3}, y_{4}\right)=(1,1,0,1)$ and, applying Theorem 2.2, we get a bilinear solution of (2.20) which, on substituting $r=-m / 18, s=-n / 36$, may be written as follows:

$$
\begin{aligned}
& y_{1}=(2 p+2 q) m+(p-2 q) n, \\
& y_{2}=(-p+2 q) m+(p+q) n, \\
& y_{3}=p m+q n, \\
& y_{4}=-p n+2 q m,
\end{aligned}
$$

where $p, q, m$ and $n$ are arbitrary parameters. Now, using the relations (2.19), we get the bilinear solution (1.5) of equation (1.4).

We note here that the complete solution of equation (1.4), obtained by the usual method as described in [1, page 432], is as follows:

$$
\begin{aligned}
& x_{1}=16 p q+26 p r, \\
& x_{2}=540 p^{2}+152 p q+304 p r-16 q^{2}-70 q r-70 r^{2}, \\
& x_{3}=-270 p^{2}-76 p q-152 p r+16 q^{2}+64 q r+61 r^{2}, \\
& x_{4}=-270 p^{2}-76 p q-152 p r+16 q^{2}+48 q r+35 r^{2},
\end{aligned}
$$

where $p, q$ and $r$ are arbitrary parameters. The bilinear solution (1.5) is also a complete solution and is clearly much simpler than the complete solution (2.22).

\section{A necessary condition for the solvability of a pair of} quadratic equations. In this section, we consider the solvability of a pair of simultaneous quadratic diophantine equations in four variables. Naturally, each of the two equations must be individually solvable for otherwise, the question of their having a common solution does not arise. We first prove a theorem that gives a necessary, although not sufficient, condition for the solvability of such equations, and then we describe a method of obtaining all integer solutions when this condition is satisfied. 
Theorem 3.1. A necessary condition that the simultaneous equations in four independent variables,

$$
\begin{aligned}
& Q_{1}\left(x_{1}, x_{2}, x_{3}, x_{4}\right)=0, \\
& Q_{2}\left(x_{1}, x_{2}, x_{3}, x_{4}\right)=0,
\end{aligned}
$$

have a nontrivial solution in integers is that there exists a nontrivial solution in integers of the quartic equation,

$$
\eta^{2}=f\left(\xi_{1}, \xi_{2}\right)=\left|A\left(\xi_{1}, \xi_{2}\right)\right|,
$$

where $A\left(\xi_{1}, \xi_{2}\right)$ is the matrix of the quadratic form

$$
\xi_{1} Q_{1}\left(x_{1}, x_{2}, x_{3}, x_{4}\right)+\xi_{2} Q_{2}\left(x_{1}, x_{2}, x_{3}, x_{4}\right) .
$$

Further, a necessary condition that there exist at least two distinct solutions of the simultaneous equations (3.1) and (3.2) is that there exists an integer solution of equation (3.3) with $\eta \neq 0$.

Proof. Any rational solution $\left(\xi_{1}, \xi_{2}, \eta\right)$ of (3.3) implies the existence of an integer solution $\left(k \xi_{1}, k \xi_{2}, k^{2} \eta\right)$ for a suitably chosen value of $k$, and hence, wherever required, it suffices to prove just the existence of a rational solution of (3.3).

We will first show that, when the simultaneous quadratic equations (3.1) and (3.2) have two distinct integer solutions, then (3.3) has a solution in integers with $\eta \neq 0$. Let $A_{j}, j=1,2$, be the respective matrices of the quadratic forms $Q_{j}\left(x_{i}\right), j=1,2$. If either of the determinants $\left|A_{i}\right|$, say $\left|A_{1}\right|$ is a nonzero perfect square, say $\eta_{1}^{2}$, a rational solution of equation $(3.3)$ is given by $\left(\xi_{1}, \xi_{2}, \eta\right)=\left(1,0, \eta_{1}\right)$.

Next we assume that neither $\left|A_{1}\right|$ nor $\left|A_{2}\right|$ is a perfect square. If

$$
\left(x_{1}, x_{2}, x_{3}, x_{4}\right)=\left(\alpha_{1}, \alpha_{2}, \alpha_{3}, \alpha_{4}\right)
$$

and

$$
\left(x_{1}, x_{2}, x_{3}, x_{4}\right)=\left(\beta_{1}, \beta_{2}, \beta_{3}, \beta_{4}\right)
$$

are two integer solutions of equations (3.1) and (3.2), we substitute

$$
x_{i}=\alpha_{i} m+\beta_{i} n, \quad i=1,2,3,4,
$$

into the equation

$$
\xi_{1} Q_{1}\left(x_{1}, x_{2}, x_{3}, x_{4}\right)+\xi_{2} Q_{2}\left(x_{1}, x_{2}, x_{3}, x_{4}\right)=0,
$$


whence we obtain the equation

$$
m n\left(\xi_{1} h_{1}+\xi_{2} h_{2}\right)=0,
$$

where both $h_{1}$ and $h_{2}$ are determined by $\alpha_{i}, i=1,2,3,4$, and $\beta_{i}, i=1,2,3,4$. We note that both $h_{1}$ and $h_{2}$ must be nonzero since either $h_{j}$ being 0 would imply that (3.4) is a linear solution in two independent parameters $m$ and $n$ of the corresponding equation $Q_{j}\left(x_{1}, x_{2}, x_{3}, x_{4}\right)=0$, and, in view of Theorem 2.4, the corresponding determinant $\left|A_{i}\right|$ must be a nonzero perfect square contradicting our assumption. Taking $\xi_{1}=-h_{2}$ and $\xi_{2}=h_{1}$, we observe that a linear solution of equation (3.5) in terms of two independent parameters $m$ and $n$ is given by (3.4), and hence it follows from Theorem 2.4 that, with these values of $\xi_{j}, j=1,2$, the determinant $A\left(\xi_{1}, \xi_{2}\right)$ must be a nonzero perfect square, that is, the diophantine equation (3.3) has a nontrivial solution with $\eta \neq 0$.

We will now consider the case when the simultaneous equations (3.1) and (3.2) have just one solution in integers, say

$$
\left(x_{1}, x_{2}, x_{3}, x_{4}\right)=\left(\alpha_{1}, \alpha_{2}, \alpha_{3}, \alpha_{4}\right),
$$

where we assume without loss of generality that $\alpha_{4} \neq 0$. By the invertible linear transformation defined by

$$
x_{1}=y_{1}+\alpha_{1} y_{4}, \quad x_{2}=y_{2}+\alpha_{2} y_{4}, \quad x_{3}=y_{3}+\alpha_{3} y_{4}, \quad x_{4}=\alpha_{4} y_{4},
$$

we transform these two equations to the corresponding equations

$$
Q_{j}^{\prime}\left(y_{1}, y_{2}, y_{3}, y_{4}\right)=0, \quad j=1,2,
$$

which have just one solution, namely,

$$
\left(y_{1}, y_{2}, y_{3}, y_{4}\right)=(0,0,0,1),
$$

and hence, the two quadratic forms $Q_{j}^{\prime}\left(y_{i}\right), j=1,2$ may be written as follows:

$$
\begin{aligned}
& Q_{1}^{\prime}\left(y_{1}, y_{2}, y_{3}, y_{4}\right)=y_{4} L_{1}\left(y_{1}, y_{2}, y_{3}\right)+Q_{3}\left(y_{1}, y_{2}, y_{3}\right), \\
& Q_{2}^{\prime}\left(y_{1}, y_{2}, y_{3}, y_{4}\right)=y_{4} L_{2}\left(y_{1}, y_{2}, y_{3}\right)+Q_{4}\left(y_{1}, y_{2}, y_{3}\right),
\end{aligned}
$$

where $L_{j}\left(y_{1}, y_{2}, y_{3}\right), j=1,2$, are linear forms, and $Q_{j}\left(y_{1}, y_{2}, y_{3}\right)$, $j=3,4$, are quadratic forms respectively, in the variables $y_{1}, y_{2}, y_{3}$. There are now two possibilities: 
(i) If the two linear forms $L_{j}\left(y_{1}, y_{2}, y_{3}\right), j=1,2$, are linearly dependent, there exist nonzero integers $h_{1}, h_{2}$ such that

$$
h_{1} L_{1}\left(y_{1}, y_{2}, y_{3}\right)+h_{2} L_{2}\left(y_{1}, y_{2}, y_{3}\right)=0 \text {, }
$$

and so the quadratic form

$$
h_{1} Q_{1}^{\prime}\left(y_{1}, y_{2}, y_{3}, y_{4}\right)+h_{2} Q_{2}^{\prime}\left(y_{1}, y_{2}, y_{3}, y_{4}\right)
$$

does not contain $y_{4}$ and therefore has at most three independent variables. Thus, the determinant of the matrix of this quadratic form must be 0 , and hence the determinant of the matrix of the quadratic form

$$
h_{1} Q_{1}\left(x_{1}, x_{2}, x_{3}, x_{4}\right)+h_{2} Q_{2}\left(x_{1}, x_{2}, x_{3}, x_{4}\right)
$$

is also 0 . Thus, $\left|A\left(h_{1}, h_{2}\right)\right|=0$, and $\left(\xi_{1}, \xi_{2}, \eta\right)=\left(h_{1}, h_{2}, 0\right)$ is a nontrivial solution of equation (3.3).

(ii) If the two linear forms $L_{j}\left(y_{1}, y_{2}, y_{3}\right), j=1,2$, are linearly independent, we choose a suitable arbitrary third form $L_{3}\left(y_{1}, y_{2}, y_{3}\right)$ such that the three forms $L_{j}\left(y_{1}, y_{2}, y_{3}\right), j=1,2,3$, are linearly independent, and apply the invertible linear transformation defined by $L_{j}\left(y_{1}, y_{2}, y_{3}\right)=z_{j}, j=1,2,3$, and $y_{4}=z_{4}$ to the two quadratic forms $Q_{j}^{\prime}\left(y_{i}\right), j=1,2$, to get corresponding quadratic forms $Q_{j}^{\prime \prime}\left(z_{i}\right)$, $j=1,2$, which may be written as follows:

$$
\begin{aligned}
& Q_{1}^{\prime \prime}\left(z_{i}\right)=z_{4} z_{1}+a_{1} z_{1}^{2}+a_{2} z_{2}^{2}+a_{3} z_{3}^{2}+a_{4} z_{2} z_{3}+a_{5} z_{1} z_{3}+a_{6} z_{1} z_{2}, \\
& Q_{2}^{\prime \prime}\left(z_{i}\right)=z_{4} z_{2}+b_{1} z_{1}^{2}+b_{2} z_{2}^{2}+b_{3} z_{3}^{2}+b_{4} z_{2} z_{3}+b_{5} z_{1} z_{3}+b_{6} z_{1} z_{2},
\end{aligned}
$$

where the coefficients $a_{j}, b_{j}, j=1, \ldots, 6$, can be effectively computed. The two equations $Q_{j}^{\prime \prime}\left(z_{i}\right)=0, j=1,2$, necessarily have just one solution in integers, namely,

$$
\left(z_{1}, z_{2}, z_{3}, z_{4}\right)=(0,0,0,1),
$$

and hence both $a_{3}$ and $b_{3}$ cannot be 0 .

Next we consider the equation

$$
b_{3} Q_{1}^{\prime \prime}\left(z_{i}\right)-a_{3} Q_{2}^{\prime \prime}\left(z_{i}\right)=0 .
$$

On substituting $z_{2}=z_{1}$ and cancelling out the factor $z_{1}$, this equation reduces to a linear equation and hence the equation $b_{3} Q_{1}^{\prime \prime}\left(z_{i}\right)-$ $a_{3} Q_{2}^{\prime \prime}\left(z_{i}\right)=0$ has a linear solution in two independent parameters. It 
follows that the corresponding equation

$$
b_{3} Q_{1}\left(x_{i}\right)-a_{3} Q_{2}\left(x_{i}\right)=0
$$

also has a linear solution in two independent parameters, and hence by Theorem 2.4, the determinant of the matrix of the quadratic form $b_{3} Q_{1}\left(x_{i}\right)-a_{3} Q_{2}\left(x_{i}\right)$ must be a nonzero perfect square. Thus, $\left|A\left(b_{3},-a_{3}\right)\right|$ is a nonzero perfect square and hence equation (3.3) has a nontrivial solution.

Thus, even when the simultaneous equations (3.1) and (3.2) have a single solution in integers, the diophantine equation (3.3) has a nontrivial solution in integers. This completes the proof of the theorem.

It is interesting to note that the simultaneous equations (3.1) and (3.2) may essentially have just one numerical solution, while the corresponding equation (3.3) has infinitely many solutions. As an example, the two equations,

$$
\begin{aligned}
Q_{1}\left(x_{1}, x_{2}, x_{3}, x_{4}\right)= & x_{1} x_{2}-x_{3} x_{4}=0 \\
Q_{2}\left(x_{1}, x_{2}, x_{3}, x_{4}\right)= & \left(x_{1}-x_{2}\right)^{2}+\left(x_{1}-x_{3}\right)^{2} \\
& +\left(x_{1}-x_{4}\right)^{2}=0
\end{aligned}
$$

have only one solution, namely $\left(x_{1}, x_{2}, x_{3}, x_{4}\right)=(1,1,1,1)$, while the determinant

$$
\left|A\left(\xi_{1}, \xi_{2}\right)\right|=\xi_{1}^{2}\left(\xi_{1}+2 \xi_{2}\right)\left(\xi_{1}-6 \xi_{2}\right) / 16,
$$

and hence equation (3.3) is easily seen to have infinitely many solutions.

We have noted earlier that the condition of Theorem 3.1 is not sufficient for the solvability of the two equations. This is illustrated by the two equations,

$$
\begin{aligned}
Q_{1}\left(x_{1}, x_{2}, x_{3}, x_{4}\right)= & x_{1} x_{2}-x_{3} x_{4}=0 \\
Q_{2}\left(x_{1}, x_{2}, x_{3}, x_{4}\right)= & \left(x_{1}-x_{2}\right)^{2}+\left(x_{1}-x_{3}\right)^{2} \\
& +\left(7 x_{1}-x_{4}\right)^{2}=0
\end{aligned}
$$

which clearly do not have a nontrivial solution in integers even though both are individually solvable, the determinant

$$
\left|A\left(\xi_{1}, \xi_{2}\right)\right|=\left(\xi_{1}^{4}-4 \xi_{1}^{3} \xi_{2}-204 \xi_{1}^{2} \xi_{2}^{2}-96 \xi_{1} \xi_{2}^{3}\right) / 16,
$$


and equation (3.3) has infinitely many solutions, two of them being

$$
\left(\xi_{1}, \xi_{2}, \eta\right)=(0,1,0) \text { and }\left(\xi_{1}, \xi_{2}, \eta\right)=(54,2,549) .
$$

\subsection{A method of solving a pair of simultaneous diophantine} equations in four variables. We will now use Theorem 3.1 to determine the solvability of a given pair of simultaneous diophantine equations (3.1) and (3.2) and show that the complete solution of these equations may be obtained by solving the equivalent system consisting of either (3.1) or (3.2) and the equation

$$
\xi_{1} Q_{1}\left(x_{1}, x_{2}, x_{3}, x_{4}\right)+\xi_{2} Q_{2}\left(x_{1}, x_{2}, x_{3}, x_{4}\right)=0,
$$

for appropriately chosen $\xi_{1}$ and $\xi_{2}$.

As before, let $A\left(\xi_{1}, \xi_{2}\right)$ be the matrix of the quadratic form $\xi_{1} Q_{1}\left(x_{i}\right)+\xi_{2} Q_{2}\left(x_{i}\right)$. We now consider the solvability of equation (3.3). If it has no nontrivial solutions in integers, it follows from Theorem 3.1 that the pair of equations (3.1) and (3.2) has no nontrivial solutions. As an example, when

$$
\begin{aligned}
& Q_{1}\left(x_{i}\right)=x_{1}^{2}+2 x_{2}^{2}-x_{3}^{2}-x_{4}^{2}, \\
& Q_{2}\left(x_{i}\right)=7 x_{1}^{2}+4 x_{1} x_{2}+14 x_{2}^{2}-6 x_{3}^{2}+2 x_{3} x_{4}-8 x_{4}^{2},
\end{aligned}
$$

both the equations $Q_{j}\left(x_{i}\right)=0, j=1,2$, individually have infinitely many solutions, but

$$
\left|A\left(\xi_{1}, \xi_{2}\right)\right|=32\left(\xi_{1}^{2}+14 \xi_{1} \xi_{2}+47 \xi_{2}^{2}\right)^{2},
$$

and so equation (3.3) does not have any nontrivial solutions. Hence, these simultaneous equations are not solvable.

When equation (3.3) is solvable, we will choose a suitable integer solution of (3.3), solve the corresponding equation (3.11) and use this solution to obtain the complete solution of equations (3.1) and (3.2). During this process, we may need to solve quartic equations of the type

$$
\eta^{2}=c_{0} \xi^{4}+c_{1} \xi^{3}+c_{2} \xi^{2}+c_{3} \xi+c_{4},
$$

where $c_{i}, i=1, \ldots, 4$, are rational numbers. While there is no algorithm that will give a numerical solution of an equation of type (3.13) in every case, such a solution can often be found using programs such as APECS. 
The following two subsections, based on reducibility of the quartic form $\left|A\left(\xi_{1}, \xi_{2}\right)\right|$, discuss the method further and give illustrative examples.

3.1.1. The determinant $\left|A\left(\xi_{1}, \xi_{2}\right)\right|$ has a linear factor. In this case, it is simplest to choose integer values of $\xi_{1}$ and $\xi_{2}$ such that $\left|A\left(\xi_{1}, \xi_{2}\right)\right|=0$ so that equation (3.11) has fewer than four independent variables. We now apply an invertible linear transformation $X=P Y$ to reduce this equation to the diagonal form (2.15), where one or more of the coefficients $a_{i}$ must be zero. We obtain the complete solution of this equation (including its trivial solutions!), determine the values of $x_{i}, i=1, \ldots, 4$, by using the relations $X=P Y$, substitute these values in (3.1) (or in (3.2)) and solve the resulting equation to obtain the complete solution of the simultaneous equations (3.1) and (3.2).

There are several possibilities depending on the solvability of the reduced equation (2.15). We give four examples illustrating different types of solutions that can be obtained.

For the simultaneous equations,

$$
\begin{aligned}
& Q_{1}\left(x_{i}\right)=x_{1}^{2}+5 x_{2}^{2}-4 x_{2} x_{4}-3 x_{3}^{2}+2 x_{4}^{2}=0, \\
& Q_{2}\left(x_{i}\right)=x_{1}^{2}+3 x_{2}^{2}-2 x_{2} x_{4}-2 x_{3}^{2}+x_{4}^{2}=0,
\end{aligned}
$$

we have

$$
\begin{gathered}
\left|A\left(\xi_{1}, \xi_{2}\right)\right|=-\left(\xi_{1}+\xi_{2}\right)\left(2 \xi_{1}+\xi_{2}\right)\left(3 \xi_{1}+2 \xi_{2}\right)^{2}, \\
2 Q_{1}\left(x_{i}\right)-3 Q_{2}\left(x_{i}\right)=-\left(x_{1}+x_{2}-x_{4}\right)\left(x_{1}-x_{2}+x_{4}\right),
\end{gathered}
$$

and the complete solution is given by

$$
x_{1}=\left(m^{2}-n^{2}\right), x_{2}=2 m n, x_{3}=\left(m^{2}+n^{2}\right), x_{4}=\left(m^{2}+2 m n-n^{2}\right),
$$

and

$x_{1}=\left(m^{2}-n^{2}\right), x_{2}=2 m n, x_{3}=\left(m^{2}+n^{2}\right), x_{4}=-\left(m^{2}-2 m n-n^{2}\right)$,

where $m$ and $n$ are arbitrary parameters.

As a second example, for the simultaneous equations,

$$
\begin{aligned}
Q_{1}\left(x_{i}\right)=x_{1}^{2}+ & 544 x_{1} x_{2}-320 x_{1} x_{3}-27 x_{2}^{2} \\
+ & 320 x_{2} x_{4}-x_{3}^{2}+320 x_{4}^{2}=0,
\end{aligned}
$$




$$
\begin{aligned}
Q_{2}\left(x_{i}\right)=x_{1}^{2}+ & 1088 x_{1} x_{2}-640 x_{1} x_{3}-55 x_{2}^{2} \\
+ & 640 x_{2} x_{4}-x_{3}^{2}+640 x_{4}^{2}=0
\end{aligned}
$$

we have

$$
\begin{aligned}
\left|A\left(\xi_{1}, \xi_{2}\right)\right|= & 320\left(\xi_{1}+2 \xi_{2}\right)\left(299 \xi_{1}+599 \xi_{2}\right)\left(97 \xi_{1}+193 \xi_{2}\right)^{2} \\
& 2 Q_{1}\left(x_{i}\right)-Q_{2}\left(x_{i}\right)=x_{1}^{2}+x_{2}^{2}-x_{3}^{2}
\end{aligned}
$$

and the complete solution is given by the single numerical solution $\left(x_{1}, x_{2}, x_{3}, x_{4}\right)=(3,4,5,-2)$ and the two parametric solutions

$$
\begin{aligned}
& x_{1}=-119 m^{4}-480 m^{3} n-350 m^{2} n^{2}+25 n^{4}, \\
& x_{2}=120 m^{4}+200 m^{3} n+120 m^{2} n^{2}+200 m n^{3}, \\
& x_{3}=169 m^{4}+480 m^{3} n+450 m^{2} n^{2}+25 n^{4}, \\
& x_{4}=-155 m^{4}-186 m^{3} n+300 m^{2} n^{2}+70 m n^{3}-25 n^{4},
\end{aligned}
$$

and

$$
\begin{aligned}
& x_{1}=-119 m^{4}-480 m^{3} n-350 m^{2} n^{2}+25 n^{4}, \\
& x_{2}=120 m^{4}+200 m^{3} n+120 m^{2} n^{2}+200 m n^{3}, \\
& x_{3}=169 m^{4}+480 m^{3} n+450 m^{2} n^{2}+25 n^{4}, \\
& x_{4}=35 m^{4}-14 m^{3} n-420 m^{2} n^{2}-270 m n^{3}+25 n^{4},
\end{aligned}
$$

where, as before, $m$ and $n$ are arbitrary parameters.

As a third example, for the simultaneous equations,

$$
\begin{aligned}
Q_{1}\left(x_{i}\right)=x_{1}^{2}+ & 4 x_{2}^{2}+8 x_{2} x_{3}+8 x_{2} x_{4} \\
& +5 x_{3}^{2}+16 x_{3} x_{4}+8 x_{4}^{2}=0 \\
Q_{1}\left(x_{i}\right)= & =0 \\
2 x_{1}^{2} & +5 x_{2}^{2}+8 x_{2} x_{3}+8 x_{2} x_{4} \\
& +4 x_{3}^{2}+16 x_{3} x_{4}+8 x_{4}^{2}=0
\end{aligned}
$$

we obtain just four solutions, namely $( \pm 2,0,2,-1)$ and $( \pm 2,0,2,-3)$.

Finally, the pair of quadratic equations,

$$
\begin{aligned}
& Q_{1}\left(x_{i}\right)=x_{1}^{2}+6 x_{2}^{2}+2 x_{2} x_{3}+16 x_{2} x_{4} \\
& -4 x_{3}^{2}+8 x_{3} x_{4}+16 x_{4}^{2}=0, \\
& \begin{aligned}
Q_{2}\left(x_{i}\right)=2 x_{1}^{2} & +7 x_{2}^{2}+2 x_{2} x_{3}+16 x_{2} x_{4} \\
- & 5 x_{3}^{2}+8 x_{3} x_{4}+16 x_{4}^{2}=0,
\end{aligned}
\end{aligned}
$$

have infinitely many solutions that are given by

$$
x_{1}=4\left(\xi^{2}-1\right), x_{2}=8 \xi, x_{3}=4\left(\xi^{2}+1\right), x_{4}=-\xi^{2}-4 \xi \pm 2 \eta-1,
$$


where $\xi$ and $\eta$ are related by

$$
\eta^{2}=\xi^{4}+\xi^{3}+\xi^{2}+\xi+1 .
$$

Equation (3.18) represents an elliptic curve of rank 1 with a point of infinite order being given by $(\xi, \eta)=(-1,1)$. We can thus find infinitely many solutions of (3.18) and hence also of the above pair of quadratic equations.

3.1.2. The determinant $\left|A\left(\xi_{1}, \xi_{2}\right)\right|$ does not have a linear factor. In this case, we take $\xi_{2}=\xi \xi_{1}$ and replace $\eta$ by $\eta \xi_{1}^{2}$ when (3.3) reduces to an equation of type (3.13) and we have to determine its solvability. If (3.3) has no integer solutions, Theorem 3.1 ensures that the simultaneous equations (3.1) and (3.2) have no integer solutions. If (3.3) has an integer solution, we choose integer values of $\xi_{1}$ and $\xi_{2}$ such that $\left|A\left(\xi_{1}, \xi_{2}\right)\right|$ is a perfect square, and obtain the complete solution of equation (3.11) in terms of bilinear forms. On substituting this solution in (3.1), we get a parametrized quadratic equation whose solvability depends on the solvability of another quartic equation of type (3.13). We thus determine the solvability of the simultaneous equations (3.1) and (3.2) and obtain their complete solution.

As an example, we consider the simultaneous equations,

$$
\begin{aligned}
Q_{1}\left(x_{i}\right)= & x_{1}^{2}-9 x_{2}^{2}-x_{3}^{2}+4 x_{4}^{2}=0 \\
Q_{2}\left(x_{i}\right)= & 3 x_{1}^{2}-30 x_{1} x_{2}-4 x_{1} x_{3}-9 x_{2}^{2} \\
& -12 x_{2} x_{3}-7 x_{3}^{2}+12 x_{3} x_{4}+4 x_{4}^{2}=0 .
\end{aligned}
$$

This is the pair of equations (1.6) mentioned in the introduction. Here,

$$
\left|A\left(\xi_{1}, \xi_{2}\right)\right|=36\left(\xi_{1}^{2}+6 \xi_{1} \xi_{2}+20 \xi_{2}^{2}\right)^{2},
$$

and so any linear combination of the two equations has a bilinear solution. We note that the equation $Q_{1}\left(x_{i}\right)=0$ is the same as equation (2.17), and its solution is given by (2.18). On substituting the values of $x_{i}$ given by $(2.18)$ in $Q_{2}\left(x_{i}\right)=0$ and solving the resulting equation, we obtain the complete solution of equations (3.19), as given by (1.7) and (1.8). 
As a second example, we consider the two simultaneous equations,

$$
\begin{array}{r}
Q_{1}\left(x_{i}\right)=6 x_{1}^{2}-4 x_{1} x_{2}+4 x_{2} x_{3}-36 x_{2} x_{4}-5 x_{3}^{2}-27 x_{4}^{2}=0, \\
Q_{2}\left(x_{i}\right)=11 x_{1}^{2}-8 x_{1} x_{2}+4 x_{2}^{2}+8 x_{2} x_{3}=0 \\
-72 x_{2} x_{4}-9 x_{3}^{2}-63 x_{4}^{2}=0 .
\end{array}
$$

Here, we get

$\left|A\left(\xi_{1}, \xi_{2}\right)\right|=9828 \xi_{1}^{4}+78336 \xi_{1}^{3} \xi_{2}+234360 \xi_{1}^{2} \xi_{2}^{2}+311616 \xi_{1} \xi_{2}^{3}+155268 \xi_{2}^{4}$,

and taking $\left(\xi_{1}, \xi_{2}\right)=(2,-1)$ makes $\left|A\left(\xi_{1}, \xi_{2}\right)\right|$ a perfect square so that the equation $2 Q_{1}\left(x_{i}\right)-Q_{2}\left(x_{i}\right)=0$ has a bilinear solution which is given by

$x_{1}=6 p m+6 q n, x_{2}=-3 p m+3 q n, x_{3}=6 q m+6 p n, x_{4}=2 q m-2 p n$.

On substituting these values of $x_{i}$ in either of the two equations (3.20), we get the quadratic equation

$$
\left(2 p^{2}+p q-2 q^{2}\right) m^{2}+\left(-2 p^{2}+2 p q-q^{2}\right) m n+\left(-2 p^{2}+2 p q+q^{2}\right) n^{2}=0,
$$

which has a rational solution if and only if $p, q$ are chosen such that the discriminant $20 p^{4}-16 p^{3} q-24 p^{2} q^{2}+8 p q^{3}+9 q^{4}$ becomes a perfect square. We thus obtain the following solution of the simultaneous equations (3.20):

$$
\begin{aligned}
& x_{1}=12 \xi^{3}+12 \xi^{2}+18 \xi+6 \eta \xi-24, \\
& x_{2}=-6 \xi^{3}+18 \xi^{2}+3 \xi-3 \eta \xi-12, \\
& x_{3}=24 \xi^{3}+24 \xi^{2}-36 \xi+6 \eta+6, \\
& x_{4}=-8 \xi^{3}+4 \xi+2 \eta+2,
\end{aligned}
$$

where $\xi$ and $\eta$ are related by $\eta^{2}=20 \xi^{4}-16 \xi^{3}-24 \xi^{2}+8 \xi+9$. This last quartic equation represents an elliptic curve of rank 3 , three points of infinite order being

$$
\begin{aligned}
& (\xi, \eta)=\left(\frac{1}{2}, \frac{5}{2}\right) \\
& (\xi, \eta)=\left(-\frac{1}{2}, \frac{3}{2}\right)
\end{aligned}
$$


and

$$
(\xi, \eta)=\left(-\frac{14}{95}, \frac{4897}{1805}\right)
$$

We thus get infinitely many solutions of equations (3.20), the solution arising from the first of the aforementioned three points being $\left(x_{1}, x_{2}, x_{3}, x_{4}\right)=(6,21,-24,-16)$.

As a final example, we consider the simultaneous equations,

$$
\begin{aligned}
& Q_{1}\left(x_{i}\right)=x_{1}^{2}-2 x_{1} x_{2}-9 x_{2}^{2}+3 x_{3}^{2}-4 x_{3} x_{4}+11 x_{4}^{2}=0, \\
& Q_{2}\left(x_{i}\right)=6 x_{1}^{2}-x_{1} x_{2}+x_{2}^{2}-15 x_{3}^{2}-2 x_{3} x_{4}-11 x_{4}^{2}=0,
\end{aligned}
$$

both of which individually have infinitely many solutions. Here we get $\left|A\left(\xi_{1}, \xi_{2}\right)\right|=-290 \xi_{1}^{4}+454 \xi_{1}^{3} \xi_{2}+\left(\frac{37739}{4}\right) \xi_{1}^{2} \xi_{2}^{2}-\left(\frac{20035}{2}\right) \xi_{1} \xi_{2}^{3}+943 \xi_{2}^{4}$,

and taking $\xi_{1}=4, \xi_{2}=3$ makes $\left|A\left(\xi_{1}, \xi_{2}\right)\right|$ a perfect square so that the equation $4 Q_{1}\left(x_{i}\right)+3 Q_{2}\left(x_{i}\right)=0$ has a bilinear solution, which is given by

$$
\begin{aligned}
& x_{1}=12 p m+4 q n, \\
& x_{2}=8 p m-4 q n, \\
& x_{3}=5 q m+5 p n, \\
& x_{4}=-5 q m+15 p n .
\end{aligned}
$$

On substituting these values of $x_{i}$ in either of the two equations (3.21), we get the quadratic equation

$$
\left(75 q^{2}-104 p^{2}\right) m^{2}-166 p q m n+\left(375 p^{2}-16 q^{2}\right) n^{2}=0,
$$

which has a rational solution if and only if $p, q$ are chosen such that the discriminant $400\left(390 p^{4}-229 p^{2} q^{2}+12 q^{4}\right)$ becomes a perfect square. It is readily established by elementary congruence considerations that this discriminant can never become a perfect square for integer values of $p$ and $q$. Thus, the simultaneous equations (3.21) do not have any integer solutions.

4. Applications to quartic diophantine equations. We will now use bilinear solutions of quadratic diophantine equations to obtain 
rational solutions of the quartic diophantine equation

$$
y^{2}=t^{4}+a_{1} t^{3}+a_{2} t^{2}+a_{3} t+a_{4},
$$

where the coefficients $a_{i}, i=1,2,3,4$, are rational numbers. This equation represents a quartic model of an elliptic curve and has been studied extensively. Various methods of finding rational solutions of this equation are discussed in [1, pages 639-644] and [2, pages 6970, 77-78]. However, explicit formulae have not been published for deriving new rational solutions of (4.1) starting from one or two known solutions of this equation. The reason for this seems to be that the existing methods lead to cumbersome formulae.

In subsection 4.1, we prove a theorem that gives formulae by which new rational solutions of (4.1) may be obtained starting from either a single rational solution of (4.1) or from two such solutions. In subsection 4.2 , we will show these formulae generate rational solutions of (4.1) that are much simpler than the solutions obtained by existing methods. Finally, in subsection 4.3, we show that, when two solutions of the more general quartic equation

$$
y^{2}=a_{0} t^{4}+a_{1} t^{3}+a_{2} t^{2}+a_{3} t+a_{4},
$$

are known, we can still use the theorem of subsection 4.1 to find new rational solutions of (4.2). As in the case of equation (4.1), these solutions are also much simpler than the ones obtained by existing methods.

4.1. Rational solutions of the equation $y^{2}=t^{4}+a_{1} t^{3}+a_{2} t^{2}+$ $a_{3} t+a_{4}$.

Theorem 4.1. If $\left(t_{1}, y_{1}\right)$ is any rational solution of equation (4.1) with $y_{1} \neq 0$, a new rational solution of (4.1) is given by $\left(t_{11}, y_{11}\right)$, where

$$
\begin{aligned}
t_{11}=\left\{-4\left(2 t_{1}^{2}+2 a_{1} t_{1}+a_{2}\right) y_{1}^{2}+4\left(2 t_{1}^{4}+a_{1} t_{1}^{3}-a_{3} t_{1}-2 a_{4}\right) y_{1}\right. \\
\left.+\left(4 t_{1}^{3}+3 a_{1} t_{1}^{2}+2 a_{2} t_{1}+a_{3}\right)^{2}\right\} \\
\times\left[4 y_{1}\left\{\left(4 t_{1}+a_{1}\right) y_{1}+4 t_{1}^{3}+3 a_{1} t_{1}^{2}+2 a_{2} t_{1}+a_{3}\right\}\right]^{-1}, \\
y_{11}=\left\{64 y_{1}^{6}+\left(128 t_{1}^{2}+64 a_{1} t_{1}-16 a_{1}^{2}+64 a_{2}\right) y_{1}^{5}+\left(320 t_{1}^{4}+320 a_{1} t_{1}^{3}\right.\right.
\end{aligned}
$$




$$
\begin{aligned}
& \left.+\left(96 a_{1}^{2}+64 a_{2}\right) t_{1}^{2}+64\left(a_{1} a_{2}-a_{3}\right) t_{1}-16 a_{1} a_{3}+16 a_{2}^{2}\right) y_{1}^{4} \\
& +8\left(4 t_{1}^{3}+3 a_{1} t_{1}^{2}+2 a_{2} t_{1}+a_{3}\right)\left(16 t_{1}^{3}+12 a_{1} t_{1}^{2}+3 a_{1}^{2} t_{1}\right. \\
& \left.+a_{1} a_{2}-2 a_{3}\right) y_{1}^{3}-2\left(4 t_{1}+a_{1}\right)\left(4 t_{1}^{3}+3 a_{1} t_{1}^{2}+2 a_{2} t_{1}+a_{3}\right)^{3} y_{1} \\
& \left.-\left(4 t_{1}^{3}+3 a_{1} t_{1}^{2}+2 a_{2} t_{1}+a_{3}\right)^{4}\right\} \\
& \times\left[4 y_{1}\left\{\left(4 t_{1}+a_{1}\right) y_{1}+4 t_{1}^{3}+3 a_{1} t_{1}^{2}+2 a_{2} t_{1}+a_{3}\right\}\right]^{-2},
\end{aligned}
$$

provided that

$$
\left\{\left(4 t_{1}+a_{1}\right) y_{1}+4 t_{1}^{3}+3 a_{1} t_{1}^{2}+2 a_{2} t_{1}+a_{3}\right\} \neq 0 .
$$

Further, if $\left(t_{1}, y_{1}\right)$ and $\left(t_{2}, y_{2}\right)$ are two rational solutions of equation (4.1) with $t_{1} \neq t_{2}$, a new rational solution of (4.1) is given by $\left(t_{12}, y_{12}\right)$, where

$$
\begin{aligned}
t_{12}= & \left\{-2 y_{1} y_{2}+2\left(t_{1}-t_{2}\right)\left(t_{2} y_{1}-t_{1} y_{2}\right)+a_{1}\left(t_{1}+t_{2}\right) t_{1} t_{2}\right. \\
& \left.+2 a_{2} t_{1} t_{2}+a_{3}\left(t_{1}+t_{2}\right)+2 a_{4}+2\left(t_{1}^{2}-t_{1} t_{2}+t_{2}^{2}\right) t_{1} t_{2}\right\} \\
& \times\left\{\left(t_{1}-t_{2}\right)\left(2 y_{1}-2 y_{2}+a_{1}\left(t_{1}-t_{2}\right)+2 t_{1}^{2}-2 t_{2}^{2}\right)\right\}^{-1}, \\
y_{12}=[ & -\left(y_{1}-y_{2}\right)^{4}-\left(t_{1}-t_{2}\right)\left\{\left(4 t_{1}+a_{1}\right) y_{1}-\left(4 t_{2}+a_{1}\right) y_{2}\right\}\left(y_{1}-y_{2}\right)^{2} \\
- & \left(t_{1}-t_{2}\right)^{3}\left\{\left(2 t_{1}+2 t_{2}+a_{1}\right)\left(y_{1}^{2}-y_{2}^{2}\right)-4\left(t_{1}-t_{2}\right) y_{1} y_{2}\right\} \\
+ & \left(t_{1}-t_{2}\right)^{3}\left\{\left(4 t_{1}+a_{1}\right)\left(t_{1}^{2}+2 t_{1} t_{2}+3 t_{2}^{2}+a_{1} t_{1}+2 a_{1} t_{2}+a_{2}\right) y_{1}\right. \\
& \left.\quad-\left(4 t_{2}+a_{1}\right)\left(3 t_{1}^{2}+2 t_{1} t_{2}+t_{2}^{2}+2 a_{1} t_{1}+a_{1} t_{2}+a_{2}\right) y_{2}\right\} \\
+ & \left(t_{1}-t_{2}\right)^{4}\left(t_{1}^{2}+2 t_{1} t_{2}+3 t_{2}^{2}+a_{1} t_{1}+2 a_{1} t_{2}+a_{2}\right) \\
& \left.\times\left(3 t_{1}^{2}+2 t_{1} t_{2}+t_{2}^{2}+2 a_{1} t_{1}+a_{1} t_{2}+a_{2}\right)\right] \\
& \times\left\{\left(t_{1}-t_{2}\right)\left(2 y_{1}-2 y_{2}+a_{1}\left(t_{1}-t_{2}\right)+2 t_{1}^{2}-2 t_{2}^{2}\right)\right\}^{-2},
\end{aligned}
$$

provided that $\left\{2 y_{1}-2 y_{2}+a_{1}\left(t_{1}-t_{2}\right)+2 t_{1}^{2}-2 t_{2}^{2}\right\} \neq 0$.

Proof. If $\left(t_{1}, y_{1}\right)$ is a solution of (4.1), we have

$$
a_{4}=y_{1}^{2}-\left(t_{1}^{4}+a_{1} t_{1}^{3}+a_{2} t_{1}^{2}+a_{3} t_{1}\right),
$$

and, with this value of $a_{4}$, it is readily verified using a symbolic computation software such as MAPLE that $\left(t_{11}, y_{11}\right)$ is a solution of (4.1). Similarly, if $\left(t_{1}, y_{1}\right)$ and $\left(t_{2}, y_{2}\right)$ are two solutions of equation 
(4.1), we solve the relations

$$
y_{i}^{2}=t_{i}^{4}+a_{1} t_{i}^{3}+a_{2} t_{i}^{2}+a_{3} t_{i}+a_{4}, \quad i=1,2,
$$

for $a_{3}, a_{4}$ and, using these values of $a_{3}, a_{4}$, it is readily verified by MAPLE that $\left(t_{12}, y_{12}\right)$ is a solution of (4.1).

While direct computation suffices to prove the theorem, the following more elaborate proof gives a genesis of the formulae (4.3) and (4.4).

We will first derive the formulae (4.4) that give a new solution of (4.1) starting from two known rational solutions of this equation.

We make an initial assumption that the coefficients $a_{i}$ satisfy the condition $a_{1}^{3}-4 a_{1} a_{2}+4 a_{3} \neq 0$. We will now establish a relationship between the rational solutions of equation (4.1) and the solutions of the simultaneous quadratic diophantine equations given below:

$$
\begin{array}{r}
Q_{1}\left(x_{i}\right)=x_{1}^{2}+c_{1} x_{2}^{2}+c_{2} x_{2} x_{3}+x_{2} x_{4}+c_{3} x_{3} x_{4}+c_{4} x_{4}^{2}=0, \\
Q_{2}\left(x_{i}\right)=x_{1} x_{2}-x_{3} x_{4}=0,
\end{array}
$$

where the values of $c_{j}, j=1,2,3,4$, are given below:

$$
\begin{aligned}
& c_{1}=\left(a_{1}^{2}-4 a_{2}\right) / 16, \\
& c_{2}=-\left(a_{1}^{3}-4 a_{1} a_{2}+4 a_{3}\right) / 16 \\
& c_{3}=-a_{1} / 2 \\
& c_{4}=4\left(a_{1}^{4}-4 a_{1}^{2} a_{2}+8 a_{1} a_{3}-16 a_{4}\right) /\left(a_{1}^{3}-4 a_{1} a_{2}+4 a_{3}\right)^{2} .
\end{aligned}
$$

Let $\left(\alpha_{1}, \alpha_{2}, \alpha_{3}, \alpha_{4}\right)$ be an arbitrary rational solution of the simultaneous equations (4.5) and (4.6), and let $\left[\alpha_{1}, \alpha_{2}, \alpha_{3}, \alpha_{4}\right]$, written briefly as $\bar{\alpha}$, denote the equivalence class of all rational solutions $\left(k \alpha_{1}, k \alpha_{2}, k \alpha_{3}, k \alpha_{4}\right)$, where $k \in \mathbb{Q} \backslash\{0\}$. Let $S$ be the set of all equivalence classes $\left[\alpha_{1}, \alpha_{2}, \alpha_{3}, \alpha_{4}\right]$, where $\alpha_{4} \neq 0$, and let $T$ be the set of all rational solutions $\left(t_{1}, y_{1}\right)$ of equation (4.1). We will show that there is a one-to-one correspondence between the sets $S$ and $T$.

Let $\bar{\alpha}$ be any arbitrary element of $S$. We define a mapping $\phi: S \rightarrow T$ as follows:

$$
\phi(\bar{\alpha})=\left(t_{0}, y_{0}\right)
$$

where

$$
\begin{aligned}
& t_{0}=\frac{\left(a_{1}^{3}-4 a_{1} a_{2}+4 a_{3}\right) \alpha_{2}+8 a_{1} \alpha_{4}}{16 \alpha_{4}} \\
& y_{0}=\frac{\left(a_{1}^{3}-4 a_{1} a_{2}+4 a_{3}\right)\left\{32 \alpha_{1} \alpha_{4}-\left(a_{1}^{3}-4 a_{1} a_{2}+4 a_{3}\right) \alpha_{2}^{2}-8 a_{1} \alpha_{2} \alpha_{4}\right\}}{256 \alpha_{4}^{2}} .
\end{aligned}
$$


We note that the mapping $\phi$ is well-defined since it is easily seen that the values of $t_{0}$ and $y_{0}$ are independent of the scalar factor $k$. We will now show that $\left(t_{0}, y_{0}\right)$ is indeed a solution of equation (4.1). Since $\bar{\alpha} \in S$, we have $Q_{1}\left(\alpha_{i}\right)=0$ as well as $Q_{2}\left(\alpha_{i}\right)=0$, and, on eliminating $\alpha_{3}$ from these two relations, we find that $\alpha_{1}, \alpha_{2}, \alpha_{4}$ satisfy the condition $f\left(\alpha_{1}, \alpha_{2}, \alpha_{4}\right)=0$, where

$$
\begin{aligned}
f\left(\alpha_{1}, \alpha_{2}, \alpha_{4}\right)= & \left(a_{1}^{3}-4 a_{1} a_{2}+4 a_{3}\right)^{3} \alpha_{1} \alpha_{2}^{2}-16\left(a_{1}^{3}-4 a_{1} a_{2}+4 a_{3}\right)^{2} \alpha_{2} \alpha_{4}^{2} \\
& -\left(64 a_{1}^{4}-256 a_{1}^{2} a_{2}+512 a_{1} a_{3}-1024 a_{4}\right) \alpha_{4}^{3} \\
& -\left(a_{1}^{3}-4 a_{1} a_{2}+4 a_{3}\right)^{2}\left(\alpha_{2}^{2} a_{1}^{2}-8 \alpha_{1} \alpha_{2} a_{1}\right. \\
& \left.-4 \alpha_{2}^{2} a_{2}+16 \alpha_{1}^{2}\right) \alpha_{4} .
\end{aligned}
$$

Now, using the values of $\left(t_{0}, y_{0}\right)$ given by (4.8), we find that

$$
y_{0}^{2}-\left(t_{0}^{4}+a_{1} t_{0}^{3}+a_{2} t_{0}^{2}+a_{3} t_{0}+a_{4}\right)=f\left(\alpha_{1}, \alpha_{2}, \alpha_{4}\right) /\left(1024 \alpha_{4}^{3}\right)=0 .
$$

This shows that $\left(t_{0}, y_{0}\right)$ is a solution of equation (4.1) and hence $\phi(\bar{\alpha}) \in T$.

Next, let $\left(t_{1}, y_{1}\right)$ be any arbitrary element of $T$. We define a mapping $\psi: T \rightarrow S$ as follows:

$$
\psi\left(t_{1}, y_{1}\right)=\left[\beta_{1}, \beta_{2}, \beta_{3}, \beta_{4}\right],
$$

where

$$
\begin{aligned}
& \beta_{1}=4\left(a_{1}^{3}-4 a_{1} a_{2}+4 a_{3}\right)\left(2 t_{1}^{2}+t_{1} a_{1}+2 y_{1}\right) \\
& \beta_{2}=-8\left(a_{1}^{3}-4 a_{1} a_{2}+4 a_{3}\right)\left(2 t_{1}+a_{1}\right) \\
& \beta_{3}=-32\left(2 t_{1}^{2}+t_{1} a_{1}+2 y_{1}\right)\left(2 t_{1}+a_{1}\right) \\
& \beta_{4}=\left(a_{1}^{3}-4 a_{1} a_{2}+4 a_{3}\right)^{2} .
\end{aligned}
$$

We will now show that $\left[\beta_{1}, \beta_{2}, \beta_{3}, \beta_{4}\right] \in S$. It is readily verified that $Q_{1}\left(\beta_{i}\right)=0$. Further, with the above values of $\beta_{i}$, we get

$$
\begin{aligned}
Q_{2}\left(\beta_{i}\right) & =64\left(a_{1}^{3}-4 a_{1} a_{2}+4 a_{3}\right)^{2}\left\{y_{1}^{2}-\left(t_{1}^{4}+a_{1} t_{1}^{3}+a_{2} t_{1}^{2}+a_{3} t_{1}+a_{4}\right)\right\} \\
& =0 .
\end{aligned}
$$

Thus, $\left(\beta_{1}, \beta_{2}, \beta_{3}, \beta_{4}\right)$ is a solution of the simultaneous equations (4.5) and (4.6). Finally, we note that, in view of our initial assumption, $\beta_{4} \neq 0$. This proves that

$$
\left[\beta_{1}, \beta_{2}, \beta_{3}, \beta_{4}\right] \in S
$$


With the above definitions of the mappings $\phi$ and $\psi$, it is readily established that, for any $\left(t_{1}, y_{1}\right) \in T$, we have $\phi\left(\psi\left(t_{1}, y_{1}\right)\right)=\left(t_{1}, y_{1}\right)$, and for any $\bar{\alpha} \in S$, we have $\psi(\phi(\bar{\alpha}))=\bar{\alpha}$. We have thus established a one-to-one correspondence between the two sets $S$ and $T$.

Now, let $\left(t_{1}, y_{1}\right)$ and $\left(t_{2}, y_{2}\right)$ be any two distinct elements of $T$ such that $t_{1} \neq t_{2}$. Further, let

$$
\psi\left(t_{1}, y_{1}\right)=\left[\beta_{1}, \beta_{2}, \beta_{3}, \beta_{4}\right]
$$

and

$$
\psi\left(t_{2}, y_{2}\right)=\left[\gamma_{1}, \gamma_{2}, \gamma_{3}, \gamma_{4}\right],
$$

so that $\left(\beta_{1}, \beta_{2}, \beta_{3}, \beta_{4}\right)$ and $\left(\gamma_{1}, \gamma_{2}, \gamma_{3}, \gamma_{4}\right)$ are two distinct solutions of the simultaneous equations (4.5) and (4.6). Thus, we have

$$
Q_{j}\left(\beta_{1}, \beta_{2}, \beta_{3}, \beta_{4}\right)=0, \quad j=1,2,
$$

as well as

$$
Q_{j}\left(\gamma_{1}, \gamma_{2}, \gamma_{3}, \gamma_{4}\right)=0, \quad j=1,2 .
$$

The values of $\beta_{i}, i=1,2,3,4$, are given by (4.9), while the values of $\gamma_{i}, i=1,2,3,4$, are given by analogous relations with $t_{1}, y_{1}$ being replaced by $t_{2}, y_{2}$, respectively.

We now consider the quadratic equation

$$
Q_{1}\left(x_{1}, x_{2}, x_{3}, x_{4}\right)+t Q_{2}\left(x_{1}, x_{2}, x_{3}, x_{4}\right)=0,
$$

where $t$ is some rational number. It is readily verified that the determinant $|A|$ of the matrix of the quadratic form $Q_{1}\left(x_{i}\right)+t Q_{2}\left(x_{i}\right)$ is

$$
t^{4}+a_{1} t^{3}+a_{2} t^{2}+a_{3} t+a_{4} .
$$

We will now use the solutions $\left(\beta_{1}, \beta_{2}, \beta_{3}, \beta_{4}\right)$ and $\left(\gamma_{1}, \gamma_{2}, \gamma_{3}, \gamma_{4}\right)$ of equations (4.5) and (4.6) to find a value of $t$ for which equation (4.10) has a solution in terms of linear forms in two independent variables. By Theorem 2.4, this value of $t$ will make the determinant $|A|$ a perfect square, and we will thus obtain a solution of equation (4.1).

We make the following substitution in equation (4.10):

$$
\begin{aligned}
& x_{1}=\beta_{1} m+\gamma_{1} n, \quad x_{2}=\beta_{2} m+\gamma_{2} n, \\
& x_{3}=\beta_{3} m+\gamma_{3} n, \quad x_{4}=\beta_{4} m+\gamma_{4} n .
\end{aligned}
$$


Since $Q_{j}\left(\beta_{i}\right)=0, j=1,2$ and also $Q_{j}\left(\gamma_{i}\right)=0, j=1,2$, equation (4.10) reduces to

$$
\begin{gathered}
\left\{\left(\beta_{1} \gamma_{2}+\beta_{2} \gamma_{1}-\beta_{3} \gamma_{4}-\beta_{4} \gamma_{3}\right) t+2 \beta_{1} \gamma_{1}\right. \\
+\left(2 c_{1} \beta_{2}+c_{2} \beta_{3}+\beta_{4}\right) \gamma_{2}+\left(c_{2} \beta_{2}+c_{3} \beta_{4}\right) \gamma_{3} \\
\left.+\left(\beta_{2}+c_{3} \beta_{3}+2 c_{4} \beta_{4}\right) \gamma_{4}\right\} m n=0 .
\end{gathered}
$$

We now choose a value of $t$ such that equation (4.12) is satisfied. We find, using the values of $\beta_{i}, \gamma_{i}$, and the values of the coefficients $c_{j}$, $j=1,2,3,4$, given by (4.7), that it is possible to choose such a value of $t$ when

$$
\left\{2 y_{1}-2 y_{2}+a_{1}\left(t_{1}-t_{2}\right)+2 t_{1}^{2}-2 t_{2}^{2}\right\} \neq 0 ;
$$

and that this value of $t$ is given by $t_{12}$, as defined by (4.4). Thus, when $t=t_{12}$, a solution of (4.10) is given by (4.11) in terms of two independent linear parameters $m$ and $n$. It follows from Theorem 2.4 that the determinant of the matrix of the quadratic form $Q_{1}\left(x_{i}\right)+$ $t Q_{2}\left(x_{i}\right)$, that is,

$$
t^{4}+a_{1} t^{3}+a_{2} t^{2}+a_{3} t+a_{4}
$$

must be a perfect square. Thus, $t=t_{12}$ gives a rational solution of (4.1).

Using the value of $t_{12}$ obtained above, the corresponding value of $y_{12}$ given by (4.4) was obtained by direct computation using MAPLE.

We now consider the quartic equation (4.1), when the coefficients $a_{i}$ are such that $a_{1}^{3}-4 a_{1} a_{2}+4 a_{3}=0$, so that our initial assumption does not hold. In this case, we make a change of variable by replacing $t$ by $t+m$, where the value of $m$ is suitably chosen such that coefficients of the transformed quartic equation do not satisfy the condition $a_{1}^{3}-$ $4 a_{1} a_{2}+4 a_{3}=0$. We then use the known solutions $\left(t_{1}, y_{1}\right)$ and $\left(t_{2}, y_{2}\right)$ of equation (4.1) to find corresponding known solutions of the transformed quartic equation, and use them by applying the formula just found, in order to find a new rational solution of the transformed quartic equation, eventually obtaining a new solution of the original quartic equation (4.1). This new solution is given by $\left(t_{12}, y_{12}\right)$, so that the formulae (4.4) hold even when $a_{1}^{3}-4 a_{1} a_{2}+4 a_{3}=0$. The only condition is that

$$
\left(t_{1}-t_{2}\right)\left\{2 y_{1}-2 y_{2}+a_{1}\left(t_{1}-t_{2}\right)+2 t_{1}^{2}-2 t_{2}^{2}\right\} \neq 0 .
$$


To obtain formulae (4.3), we consider the value of $t_{12}$ given by (4.4) as a continuous function of $t_{2}$ while $t_{1}$ and $y_{1}$ are fixed. We found that $\lim _{t_{2} \rightarrow t_{1}} t_{12}=t_{11}$, where the value of $t_{11}$ is given by (4.3). Direct computation now confirms that $t=t_{11}$ indeed gives a rational solution of (4.1) and also yields the value of $y_{11}$ as given by (4.3). This completes the proof.

Corollary 4.2. The quartic function of $t$ given by

$$
\left(t-a_{1}\right)\left(t-a_{2}\right)\left(t^{2}+a_{3} t+a_{4}\right)
$$

becomes a perfect square if we take $t=\left(a_{1} a_{2}-4\right) /\left(a_{1}+a_{2}+a_{3}\right)$.

This follows immediately from (4.4) by taking $\left(t_{1}, y_{1}\right)=\left(a_{1}, 0\right)$ and $\left(t_{2}, y_{2}\right)=\left(a_{2}, 0\right)$ as the two known solutions.

Corollary 4.3. The quartic function of $t$ given by

$$
\left(t-a_{1}\right)\left(t-a_{2}\right)\left(t^{2}+a_{3} t+a_{4}\right)+m^{2}
$$

becomes a perfect square if we take $t=\left(a_{1} a_{2}-2 m-4\right) /\left(a_{1}+a_{2}+a_{3}\right)$.

As in the case of Corollary 4.2, this follows immediately from (4.4) by taking $\left(t_{1}, y_{1}\right)=\left(a_{1}, m\right)$ and $\left(t_{2}, y_{2}\right)=\left(a_{2}, m\right)$ as the two known solutions.

We note that the formulae (4.4) are symmetric with respect to the two solutions $\left(t_{1}, y_{1}\right)$ and $\left(t_{2}, y_{2}\right)$ and so interchanging these solutions in formulae (4.4) does not yield a new solution. Further, since the existence of any solution $\left(t_{1}, y_{1}\right)$ of equation (4.1) automatically implies the existence of the solution $\left(t_{1},-y_{1}\right)$, we can choose either sign for $y_{1}$ and $y_{2}$ in (4.3) and (4.4) and, in general, obtain two new solutions of (4.1) starting from a single known solution and four new solutions starting from two known solutions. There are, of course, examples when we get fewer solutions, or in exceptional situations, no new solutions.

4.2. A comparison with existing methods. We will now compare the results of Theorem 4.1 with the existing methods of obtaining rational solutions of equation (4.1). 
Fermat gave a procedure (as quoted by Dickson [1, page 639]) of finding a rational solution of (4.1) under fairly general conditions as well as of finding a new rational solution of (4.2), starting from a known solution. The procedure can often, though not always, be repeated any number of times to obtain infinitely many solutions of (4.1). The solution of (4.1) obtained by Fermat's method is given by a rational function of the coefficients $a_{i}$ with the numerator being a polynomial of degree 4 in $a_{i}$ and the denominator being a polynomial of degree 3 in $a_{i}$. Therefore, repetitions of the process rapidly generate rational solutions of great height.

Mordell [2, page 70], gives a substitution based on two known rational solutions $\left(t_{1}, y_{1}\right)$ and $\left(t_{2}, y_{2}\right)$ of (4.2), and this yields several new solutions of (4.2). While the resulting new rational solutions are not given explicitly, they can be worked out using a software such as MAPLE and are given by rational functions of $\left(t_{1}, y_{1}\right)$ and $\left(t_{2}, y_{2}\right)$. For any given quartic equation of type (4.1), if we take $t_{2}=O\left(t_{1}\right)$, Mordell's method gives one set of solutions in which the numerator is of order $t_{1}^{9}$, while the denominator is of order $t_{1}^{8}$, and another set of solutions in which the numerator is of $O\left(t_{1}^{17}\right)$, while the denominator is of $O\left(t_{1}^{16}\right)$.

We can also apply a birational transformation to the quartic equation (4.1) to transform it to a cubic equation (see [2, page 77]), obtain two rational solutions of this cubic equation corresponding to two known solutions of (4.1), use these two solutions to obtain another rational solution of the cubic equation and, finally, find the corresponding solution of (4.1) by using the birational transformation. This leads to very complicated formulae where, again, assuming that $t_{2}=O\left(t_{1}\right)$, we find that the numerator of the new solution is of the order of $t_{1}^{9}$, while the denominator is of order of $t_{1}^{8}$.

As compared to the above, for any given quartic equation of type (4.1), if we take $t_{2}=O\left(t_{1}\right)$, the numerator of the solution (4.4) given by Theorem 4.1 is of order $t_{1}^{4}$, while the denominator is of order $t_{1}^{3}$.

We now consider a numerical example. The quartic equation

$$
y^{2}=t^{4}-7 t^{3}-3 t^{2}+48 t-35,
$$

has the solutions

$$
(t, y)=(1, \pm 2) \quad \text { and } \quad(t, y)=(2, \pm 3) .
$$


On applying formulae (4.4) to these solutions, we get four values of $t$ that make the right-hand side of (4.13) a perfect square, namely, $18,-30 / 11,58 / 9$ and $-22 / 3$, while applying formulae (4.3) to the known solutions individually, we get four additional such values of $t$, namely, $1001 / 152,-729 / 248,-121 / 39$ and $421 / 57$. As a comparison, we now find the values of $t$ that make the right-hand side of (4.13) a perfect square by various other methods. Direct application of Fermat's method gives $t=-5961 / 344$, while using the known solutions and applying other classical methods as discussed in [1, pages 639-640], we obtain the following values of $t$ : $221 / 97,141 / 65,142 / 109$ and 70/61. Repetition of the procedures yields solutions of greater height.

The substitution mentioned by Mordell leads to the following values of $t$ :

$$
\frac{1002}{425}, \frac{2202}{2009}, \frac{169}{112}, \frac{1201}{520}, \frac{1547585}{829473}, \frac{52238}{61093} .
$$

For a further comparison, we used the software APECS to determine rational solutions of (4.1). First, we reduced the quartic equation (4.1), by a birational transformation, to the Weierstrass minimal form,

$$
Y^{2}=X^{3}-199 X-29 .
$$

Equation (4.14) represents an elliptic curve of rank 4, a basis for this elliptic curve being given by the four points $P_{1}=(-1,13)$, $P_{2}=(-5,29), P_{3}=(-13,19)$ and $P_{4}=(15,19)$. The four values of $t$ corresponding to these four points are as follows: 70/61, 6/7, 142/109 and 18 , while the four values of $t$ corresponding to the points $2 P_{i}, i=$ 1, 2, 3, 4, are as follows: 877941/130585, 11365/377, -2703129/708263 and $-21785 / 4807$. Adding two of the four points $P_{i}$ at a time, we get six new points on the elliptic curve (4.14), and the values of $t$ corresponding to these six points are as follows:

$$
\frac{223}{31},-\frac{5961}{344}, \frac{7401}{4112},-\frac{2287}{680}, \frac{55}{24}, \frac{141}{65} .
$$

On observing all the solutions obtained by various methods, it seems to be quite clear that the solutions obtained by the formulae of Theorem 4.1 compare very well with the existing methods. The comparison is even better when the coefficients $a_{i}$ of the quartic function are given in parametric terms, and one or two solutions of 
(4.1) are known, for then the formulae of Theorem 4.1 easily give new solutions of lower degree as compared to other methods.

4.3. Rational solutions of the equation $y^{2}=a_{0} t^{4}+a_{1} t^{3}+a_{2} t^{2}+$ $a_{3} t+a_{4}$. We now show that it is possible to use Theorem 4.1, even in the case of the more general quartic equation (4.2), provided we know two solutions $\left(t_{1}, y_{1}\right)$ and $\left(t_{2}, y_{2}\right)$ of $(4.2)$ such that $t_{1} \neq t_{2}$. In fact, on substituting $t=s+t_{1}$ in (4.2), we get

$$
y^{2}=b_{0} s^{4}+b_{1} s^{3}+b_{2} s^{2}+b_{3} s+y_{1}^{2},
$$

where the coefficients $b_{j}$ are determined by the coefficients $a_{i}$ and $t_{1}$, and now the further substitutions $y=y_{1} Y / r^{2}, s=1 / r$, yield an equation of the type

$$
Y^{2}=r^{4}+c_{1} r^{3}+c_{2} r^{2}+c_{3} r+c_{4},
$$

for which we can readily find a solution corresponding to the known solution $\left(t_{2}, y_{2}\right)$ of $(4.2)$. We have thus transformed equation (4.2) to an equation of type (4.1) with a known solution and can now apply the theorem. We illustrate the method by an example.

Consider the equation,

$$
y^{2}=2 t^{4}+3 t^{3}+7 t^{2}-207 t+379,
$$

for which two known solutions are $(2,7)$ and $(3,8)$. The substitution $t=s+2$ reduces the equation to

$$
y^{2}=2 s^{4}+19 s^{3}+73 s^{2}-79 s+49,
$$

and the further substitutions $y=7 Y / r^{2}, s=1 / r$, yield the quartic equation

$$
Y^{2}=r^{4}-(79 / 49) r^{3}+(73 / 49) r^{2}+(19 / 49) r+2 / 49
$$

A solution of the quartic equation (4.17), corresponding to the known solution $(3,8)$ of equation $(4.15)$ is $(1,8 / 7)$, and now, using formula (4.3), we get two solutions of (4.17), leading to two solutions of (4.15) given by $t=122 / 17$ and $t=1754 / 809$.

Following the classical method, after obtaining equation (4.16), we would write

$$
y=7-(79 / 14) s+(8067 / 2744) s^{2},
$$


so that the constant term and the coefficients of $s$ and $s^{2}$ on both sides become equal, and this gives a solution of (4.16) with $s=$ $9582440 / 1219937$, and we finally get a solution of (4.16) given by $t=12022314 / 1219937$.

This example shows that, even in the case of the more general equation (4.2), applying Theorem 4.1 yields simpler solutions as compared to the classical methods.

\section{REFERENCES}

1. L.E. Dickson, History of theory of numbers, Volume 2, Chelsea Publishing Company, New York, 1992.

2. L.J. Mordell, Diophantine equations, Academic Press, London, 1969.

3. W. Sierpinski, Elementary theory of numbers, PWN-Polish Scientific Publishers, Warszawa, 1987.

13/4 A, Clay Square, Lucknow 226001, India

Email address: ajaic203@yahoo.com 